\title{
Efficacy and safety of hypertonic saline solutions fluid resuscitation on hypovolemic shock: A systematic review and meta-analysis of randomized controlled trials
}

\author{
Kamil Safiejko ${ }^{1}$, Jacek Smereka ${ }^{2,3}$, Michal Pruc ${ }^{3}$, Jerzy R. Ladny ${ }^{3,4}$, \\ Milosz J. Jaguszewski ${ }^{5}$, Krzysztof J. Filipiak ${ }^{6}$, Ruslan Yakubtsevich ${ }^{7}$, Lukasz Szarpak ${ }^{1,3,8}$ \\ ${ }^{1}$ Maria Sklodowska-Curie Bialystok Oncology Center, Bialystok, Poland \\ ${ }^{2}$ Department of Emergency Medical Service, Wroclaw Medical University, Wroclaw, Poland \\ ${ }^{3}$ Polish Society of Disaster Medicine, Warsaw, Poland \\ ${ }^{4}$ Clinic of Emergency Medicine and Disaster, Medical University Bialystok, Poland \\ ${ }^{5}$ First Department of Cardiology, Medical University of Gdansk, Poland \\ ${ }^{6}$ First Chair and Department of Cardiology, Medical University of Warsaw, Poland \\ ${ }^{7}$ Department of Anesthesiology and Intensive Care, Grodno State Medical University, Grodno, Belarus \\ ${ }^{8}$ Maria Sklodowska-Curie Medical Academy, Warsaw, Poland
}

This paper was guest edited by Prof. Togay Evrin

\begin{abstract}
Background: Fluid resuscitation is a fundamental intervention in patients with hypovolemic shock resulting from trauma. Appropriate fluid resuscitation in trauma patients could reduce organ failure, until blood components are available, and hemorrhage is controlled. We conducted a systematic review and meta-analysis assessing the effect of hypertonic saline/dextran or hypertonic saline for fluid resuscitation on patient outcomes restricted to adults with hypovolemic shock.

Methods: We conducted a search of electronic information sources, including PubMed, Embase, Web of Science, Cochrane library and bibliographic reference lists to identify all randomized controlled trials (RCTs) investigating outcomes of crystalloids versus colloids in patients with hypovolemic shock. We calculated the risk ratio $(R R)$ or mean difference $(M D)$ of groups using fixed or random-effect models.

Results: Fifteen studies including 3264 patients met our inclusion criteria. Survival to hospital discharge rate between research groups varied and amounted to $71.2 \%$ in hypertonic saline/dextran group vs. $68.4 \%$ for isotonic/normotonic fluid (normal saline) solutions (odds ratio [OR] $=1.19 ; 95 \%$ confidence interval [CI] 0.97-1.45; $I^{2}=48 \% ; p=0.09$ ). 28- to 30-days survival rate for hypertonic fluid solutions was $72.8 \%$ survivable, while in the case of isotonic fluid (normal saline) $-71.4 \%$ $\left(O R=1.13 ; 95 \%\right.$ CI 0.75-1.70; $\left.I^{2}=43 \% ; p=0.56\right)$.

Conclusions: This systematic review and meta-analysis, which included only evidence from RCTs hypertonic saline/dextran or hypertonic saline compared with isotonic fluid did not result in superior 28- to 30-day survival as well as in survival to hospital discharge. However, patients with hypotension who received resuscitation with hypertonic saline/dextran had less overall mortality as patients who received conventional fluid. (Cardiol J 2022; 29, 6: 966-977)
\end{abstract}

Key words: fluid resuscitation, hypovolemic shock, trauma, injury, hypertonic saline, normal saline, treatment, crystalloid, colloid fluid

Address for correspondence: Lukasz Szarpak, Assoc. Prof. PhD, MBA, Bialystok Oncology Center, ul. Ogrodowa 12, 15-027 Białystok, Poland, tel: +48 500186225, e-mail: lukasz.szarpak@gmail.com

Received: 28.08.2020 Accepted: 2.09.2020 Early publication date: 22.10.2020

This article is available in open access under Creative Common Attribution-Non-Commercial-No Derivatives 4.0 International (CC BY-NC-ND 4.0) license, allowing to download articles and share them with others as long as they credit the authors and the publisher, but without permission to change them in any way or use them commercially. 


\section{Introduction}

Fluid resuscitation is a fundamental intervention in patients with hypovolemic shock resulting from trauma. The main purpose of undertaking fluid therapy is to stabilize post-traumatic circulation disorders [1, 2]. Baroreceptor-mediated, catecholamine-induced venoconstriction acts on the venous capacitance system to increase venous return and maintain cardiac output, moreover the renin-angiotensin-aldosterone and adrenocortical systems produces an antidiuretic response to retain water [3]. The hypovolemic shock caused by acute hemorrhage occurs when intravascular volume loss exceeds the capacity of these compensatory mechanisms, resulting in the compromise of vital organ perfusion [4].

Advanced trauma life support recommends the prehospital assessment of a patient's circulation status and to resuscitate with intravenous fluids in patients with obvious hemorrhage or systolic blood pressure (SBP) below $90 \mathrm{mmHg}$ [5]. A particularly important problem of fluid therapy is observed in patients with multi-organ injuries, including those affecting the central nervous system, which is extremely susceptible to osmolality changes. For this reason, hypotonic solutions that increase intracerebral water and exacerbate post-traumatic brain edema are not recommended in patients treated for head injuries [6, 7]. As indicated by Reddy et al. [8] most infusion solutions exhibit hypoosmotic effects, since only some components of crystalline solutions are active in plasma and their osmotic coefficient is 0.92 .

Crystalloid is a broad term that can encompass many different types of solutions from hypertonic normal saline (NS) to lactated Ringer's solution to $5 \%$ dextrose and half NS. $0.9 \%$ sodium chloride (NS) is one of the most frequently administered solutions. It is also the basis for the preparation of many colloids, including hypertonic saline/dextran, human albumins or gelatins.

When infused, crystalloids with a sodium concentration close to that of intravascular fluid $(140 \mathrm{mmol} / \mathrm{L})$ produce a transient increase in intravascular volume before equilibrating with the extracellular fluid. Crystalloids can be used either as resuscitation fluids (to increase or maintain intravascular volume) or as maintenance fluids (to maintain hydration and basic electrolyte balance) in persons unable to tolerate enteral administration of fluid [9]. As indicated by the meta-analysis published by Safiejko et al. [10] hypotensive fluid resuscitation significantly reduced the mortality of traumatic hemorrhagic shock patients. Rapid administration of a large volume will cause hyperchloremic metabolic acidosis. This is because in the case of a standard $0.9 \% \mathrm{NaCl}$ strong ion difference between $0.9 \% \mathrm{NaCl}$ fluid solution and plasma (respectively 0 vs. $40 \mathrm{mEq} / \mathrm{L}$ ). Therefore, it is important to know the effect of using both isotonic vs. hypertonic fluid solutions during fluid resuscitation of trauma patients.

The present study is a systematic review and meta-analysis assessing the effect of hypertonic saline fluid resuscitation on patient outcomes restricted to adults with hypovolemic shock.

\section{Methods}

This review was conducted and presented according to Preferred Reporting Items for Systematic Reviews and Meta-Analyses (PRISMA) statement standards [11]. We did not publish a prior protocol for this review.

\section{Literature searches}

A computerized literature search was conducted from the PubMed, Embase, Web of Science, and the Cochrane library data bases from their inception to August $20^{\text {th }} 2020$. In addition to the reference lists of the selected articles they were hand-searched to identify additional relevant reports. Google Scholar and other Internet search engines were also used to search for additional information.

The search terms comprised the followings: (crystalloid* OR normal saline* OR saline OR Ringers OR Ringer's OR Hartmanns OR Hartmann's OR hypertonic OR 7.5\% saline OR $\mathrm{NaCl}$ OR sodium chloride) AND (Emergency medicine OR Emergency treatment OR Emergency department OR Emergency room OR Emergency medical service OR EMS OR Hemorrhagic shock OR Hypovolemic shock OR trauma).

\section{Selection and exclusion criteria}

Two reviewers (K.S. and A.S.) independently screened the titles and abstracts of all citations retrieved during the literature search based on inclusion criteria. Disagreements were resolved through discussion until consensus was reached. Inclusive criteria: (a) Research types: randomized controlled trials (RCTs) and quasi-randomized trials; (b) Research subjects: human studies involved adult patients needing fluid resuscitation were involved in the meta-analysis. Also included were studies which were in preprint. Observational stud- 
ies, case-control studies, non-trials conducted on simulated models, editorials, reviews, guidelines, meta-analysis, and theoretical models were excluded from the review. The search was limited to English language studies and adult patients needing fluid resuscitation. The data were recorded using Review Manager.

\section{Data extraction}

Two authors (K.S. and J.R.L.) independently reviewed all identified titles and abstracts against the prespecified eligibility criteria using a standardized form piloted before the study. The reviewers then independently evaluated the full texts of the selected articles, applied the selection criteria to them, and compared decisions for all the included and excluded studies. Disagreements were resolved by discussion with the other authors (J.S.). The duplicate publications of the same trial were excluded from the present study.

The clinical data were extracted as the following: the name of the first author, the year published, the country of the author, the types of study design, the number of patients, type of fluid infused, and follow-up time. The primary outcome herein, was survival to hospital discharge or at 28 to 30 days. Other mortality periods were also extracted as defined by the authors.

\section{Outcome measures}

The primary endpoint was short-term survival (hospital discharge or 28 to 30 days). Secondary outcomes included long-term mortality ( $\geq 3$ months), 24-hour mortality, overall mortality, adverse outcome, length of stay in an intensive care unit and hospital, laboratory parameters at patient admission, the Glasgow Outcome Scale Extended score.

\section{Risk of bias assessment}

Two authors independently assessed the methodological quality and risk of bias of the included articles using the method outlined in the Cochrane Collaboration Handbook for Systematic Reviews of Interventions [12]. Risk of bias was assessed as high, low, and unclear for each of selection bias: random sequence generation, allocation concealment, blinding of participants and personnel, blinding of outcome assessment, incomplete outcome data, selective reporting, and other biases. The review authors' judgments about each risk of bias item are provided in Supplementary Digital File 1.

\section{Statistical analysis}

All analyses were performed by the Review Manager Version 5.4. (The Cochrane Collaboration, Oxford, Copenhagen, Denmark). Dichotomous data were presented as risk ratios using the Mantel-Haenszel method. Continuous data were presented as means with standard deviations and analyzed using the inverse variance. The random-effects model was used for $\mathrm{I}^{2}>50 \%$; otherwise, the fixed effects model was employed. When continuous data were presented as medians with ranges, the data were converted for inclusion into the meta-analysis using the method described by Hozo et al. [13]. Heterogeneity among the studies was assessed using the Cochran $\mathrm{Q}$ test $\left(\chi^{2}\right)$. Inconsistency was quantified by calculating $\mathrm{I}^{2}$ and was interpreted using the following guide: no heterogeneity, $\mathrm{I}^{2}=0-25 \%$; moderate heterogeneity, $\mathrm{I}^{2}=25-50 \%$; large heterogeneity, $\mathrm{I}^{2}=50-75 \%$; extreme heterogeneity, $\mathrm{I}^{2}=75-100 \%$. Where appropriate, subgroup analyses were performed based on the study design and methodological quality.

\section{Role of the funding source}

There was no funding source for this study. The corresponding author had full access to all the data in the study and had final responsibility for the decision to submit for publication.

\section{Results}

\section{Study selection}

The comprehensive search yielded 1271 potentially relevant articles; after exclusion of duplicates and assessment of titles and/or abstracts, 43 articles were chosen for complete review. Finally, 15 studies including 3264 patients met our inclusion criteria, published between 1987 and 2011 [14-28]. Figure 1 shows the flow of studies through the review.

\section{Characteristics of included studies}

The studies comprised a total of 3264 participants, of whom $54.9 \%$ were exposed to hypertonic saline solutions (Table 1 ).

All studies were RCTs. Eight studies were conducted in the United States of America [15, 18-20, 24-26], two in Brazil [27, 28], two in Canada $[21,22]$, one in the United Kingdom [14], and one in Australia [17]. One study was multi-county [16]. In general, the studies were judged as being of good quality. Supplementary Digital File 1 presents 


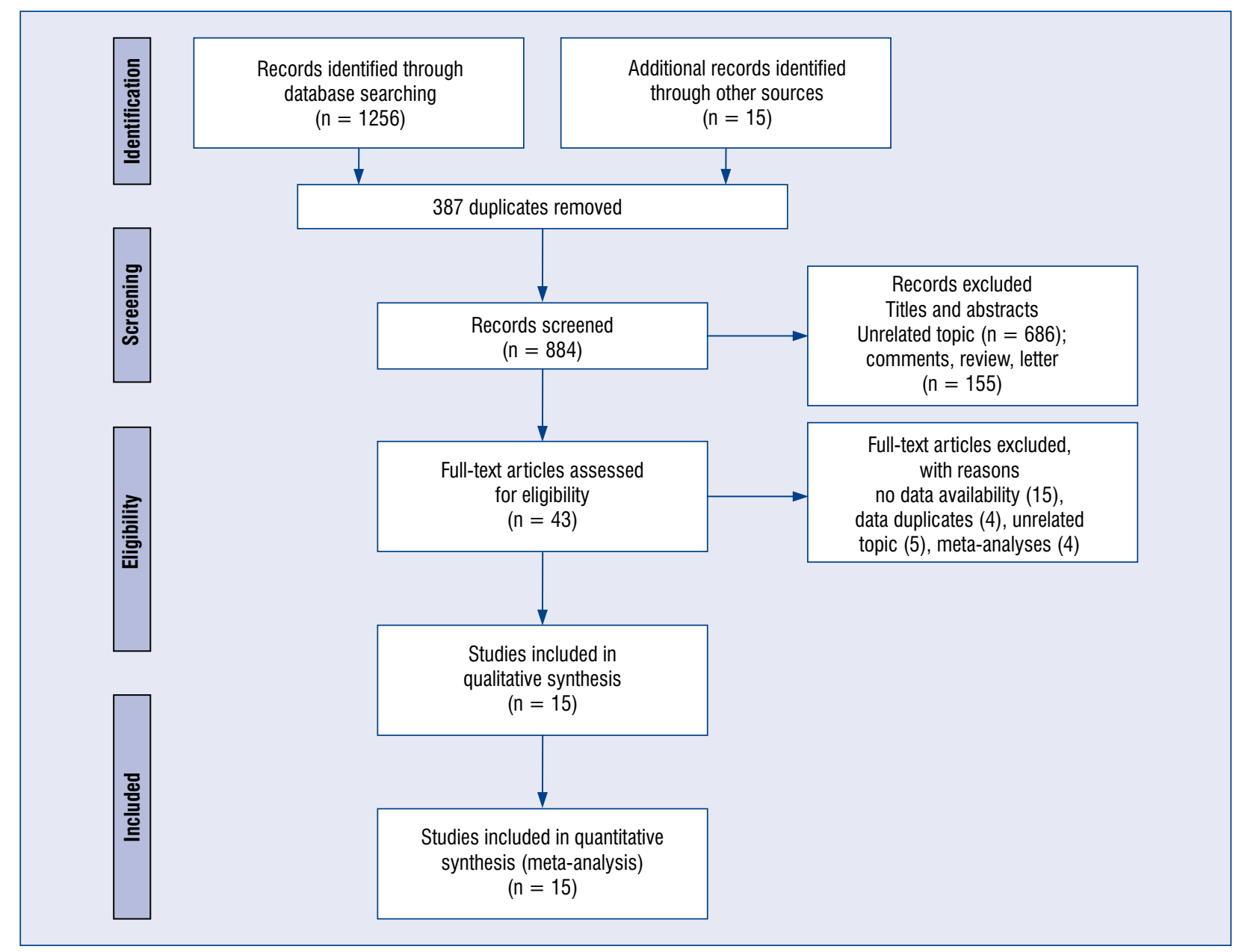

Figure 1. Flow diagram showing stages of database searching and study selection as per PRISMA guidelines.

inclusion and exclusion criteria, primary outcome as well as 28- to 30-day survival rate with an odds ratio $(\mathrm{OR})(95 \%$ confidence interval $[\mathrm{CI}])$.

\section{Primary endpoint}

In summary, 9 studies reported survival to hospital discharge including 2081 patients [16, 17, 21-27]. Survival to hospital discharge rate between research groups varied and amounted to $71.2 \%$ in hypertonic saline/dextran (HSD) group vs. $68.4 \%$ for isotonic fluid (NS) solutions $(\mathrm{OR}=1.19 ; 95 \%$ CI $0.97-1.45 ; \mathrm{I}^{2}=48 \%$; $\mathrm{p}=0.09$ ).

Subgroup analysis is shown in Figure 2. Eight studies reported a comparison between HSD and control group [16, 21-27]. The differences in terms of survival to hospital discharge were not significant and were respectively $72.8 \%$ vs. $72.3 \%$ $\left(\mathrm{OR}=1.13 ; 95 \%\right.$ CI $0.89-1.44 ; \mathrm{I}^{2}=36 \%$; $\left.\mathrm{p}=0.30\right)$. In turn, 4 studies $[16,17,25,27]$ reported analyzed comparison between hypertonic saline and isotonic saline $(68.8 \%$ vs. $68.1 \%$, respectively; $\mathrm{OR}=1.10$; $95 \%$ CI $0.83-1.44 ; \mathrm{I}^{2}=0 \% ; \mathrm{p}=0.51$ ).

28- to 30-day survival rate was reported by 5 studies [15, 16, 19, 21, 28]. Pooled analysis showed that the use of hypertonic fluid solutions was $72.8 \%$ survivable, while in the case of isotonic fluid (NS) $-71.4 \%(\mathrm{OR}=1.13 ; 95 \%$ CI $0.75-1.70$; $\mathrm{I}^{2}=43 \% ; \mathrm{p}=0.56$ ).

As shown in Figure 3 in the HSD subgroup, 4 studies indicated that hypertonic/dextran solutions infusion $[15,16,19,21]$ was associated with a survival rate of $72.6 \%$ and NS with $72.8 \%(\mathrm{OR}=$ $=1.06 ; 95 \%$ CI $\left.0.64-1.77 ; \mathrm{I}^{2}=56 \% ; \mathrm{p}=0.81\right)$. Analysis in the subgroup where the infusion of hypertonic saline vs. isotonic saline $[16,28]$ was used showed survival at the level of $73.1 \%$ vs. $71.9 \%$, respectively $(\mathrm{OR}=1.14 ; 95 \%$ CI $0.71-1.83$; $\mathrm{I}^{2}=49 \% ; \mathrm{p}=0.59$ ). 
Table 1. Characteristics of included studies.

\begin{tabular}{|c|c|c|c|c|c|c|c|c|c|}
\hline \multirow[t]{2}{*}{ Study } & \multirow[t]{2}{*}{ Country } & \multirow[t]{2}{*}{$\begin{array}{l}\text { Study } \\
\text { design }\end{array}$} & \multirow[t]{2}{*}{ Intervention } & \multicolumn{3}{|c|}{$\begin{array}{l}\text { Hyper-saline } \\
\text { group }\end{array}$} & \multicolumn{3}{|c|}{$\begin{array}{l}\text { Control } \\
\text { group }\end{array}$} \\
\hline & & & & $\mathbf{N}$ & Age & Males & $\mathbf{N}$ & Age & Males \\
\hline $\begin{array}{l}\text { Alpar et al. } \\
2004\end{array}$ & UK & RCT & $\begin{array}{l}\text { Patients randomized to receive } \\
\text { HSD or Hartmann's. HSD infused } \\
\text { at a dose of } 4 \mathrm{~mL} / \mathrm{kg} \text { or maximum } \\
250 \mathrm{~mL} \text {, with further fluid resus- } \\
\text { citation with Hartmann's or blood } \\
\text { transfusion. Average volume } \\
\text { infused: HSD group: } 4.5 \mathrm{~L} \text {, } \\
\text { Hartmann's group: } 6.5 \mathrm{~L}\end{array}$ & 90 & $\begin{array}{l}34.3 \pm \\
\pm 11.3\end{array}$ & NS & 90 & $\begin{array}{c}33.5 \pm \\
\pm 11\end{array}$ & ns \\
\hline $\begin{array}{l}\text { Bulger et al. } \\
2008\end{array}$ & USA & $\begin{array}{l}\text { Double- } \\
\text {-blind RCT }\end{array}$ & $\begin{array}{l}\text { Prehospital resuscitation with } \\
250 \mathrm{~mL} \text { either HSD or Ringer's } \\
\text { lactate. Additional ongoing resus- } \\
\text { citation with Ringer's lactate only }\end{array}$ & 110 & $\begin{array}{l}41 \pm \\
\pm 18\end{array}$ & $\begin{array}{c}69 \\
(62.7 \%)\end{array}$ & 99 & $\begin{array}{l}38 \pm \\
\pm 19\end{array}$ & $\begin{array}{c}68 \\
(68.7 \%)\end{array}$ \\
\hline $\begin{array}{l}\text { Bulger et al. } \\
2011\end{array}$ & $\begin{array}{l}\text { Multi- } \\
\text {-country }\end{array}$ & $\begin{array}{l}\text { Multi- } \\
\text {-center } \\
\text { double- } \\
\text {-blind RCT }\end{array}$ & $\begin{array}{c}\text { Patients randomized to receive } \\
\text { a } 250-\mathrm{mL} \text { bolus of either } 7.5 \% \mathrm{HS}, \\
7.5 \% \mathrm{HSD} 70 \text { or NS, in prehospi- } \\
\text { tal setting }\end{array}$ & 476 & $\begin{array}{l}37.2 \pm \\
\pm 16.7\end{array}$ & $\begin{array}{c}375 \\
(78.8 \%)\end{array}$ & 376 & $\begin{array}{l}36.2 \pm \\
\pm 16.4\end{array}$ & $\begin{array}{c}291 \\
(77.4 \%)\end{array}$ \\
\hline $\begin{array}{l}\text { Cooper et } \\
\text { al. } 2004\end{array}$ & Australia & $\begin{array}{l}\text { Double- } \\
\text {-blind RCT }\end{array}$ & $\begin{array}{l}\text { Patients randomized to receive } \\
\text { a } 250 \mathrm{~mL} \text { bolus of either } 7.5 \% \\
\text { saline or Ringer's lactate solution }\end{array}$ & 114 & $\begin{array}{l}38 \pm \\
\pm 19\end{array}$ & $\begin{array}{c}75 \\
(65.8 \%)\end{array}$ & 115 & $\begin{array}{l}37 \pm \\
\pm 19\end{array}$ & $\begin{array}{c}76 \\
(66.1 \%)\end{array}$ \\
\hline $\begin{array}{l}\text { Holcroft et } \\
\text { al. } 1987\end{array}$ & USA & $\mathrm{RCT}$ & $\begin{array}{l}\text { Patients randomized to receive } \\
\text { a } 3 \% \mathrm{NaCl}(1028 \mathrm{mOsm} / \mathrm{kg}, \\
4 \mathrm{~mL} / \mathrm{kg} \text { ) or lactated Ringer's } \\
\text { solution }(12 \mathrm{~mL} / \mathrm{kg})\end{array}$ & 10 & $\begin{array}{l}36 \pm \\
\pm 13\end{array}$ & $\begin{array}{c}9 \\
(90.0 \%)\end{array}$ & 10 & $\begin{array}{l}36 \pm \\
\pm 21\end{array}$ & $\begin{array}{c}9 \\
(90.0 \%)\end{array}$ \\
\hline $\begin{array}{l}\text { Holcroft et } \\
\text { al. } 1989\end{array}$ & USA & $\mathrm{RCT}$ & $\begin{array}{l}\text { Patients randomized to receive } \\
\text { a } 3 \% \mathrm{NaCl}(1028 \mathrm{mOsm} / \mathrm{kg}, \\
4 \mathrm{~mL} / \mathrm{kg} \text { ) or lactated Ringer's } \\
\text { solution }(12 \mathrm{~mL} / \mathrm{kg})\end{array}$ & 29 & $\begin{array}{c}38 \pm \\
\pm 15.6\end{array}$ & $\begin{array}{c}23 \\
(79.3 \%)\end{array}$ & 31 & $\begin{array}{l}38 \pm \\
\pm 19\end{array}$ & $\begin{array}{c}26 \\
(83.9 \%)\end{array}$ \\
\hline $\begin{array}{l}\text { Mattox et } \\
\text { al. } 1991\end{array}$ & USA & $\begin{array}{l}\text { Multi- } \\
\text {-center } \\
\text { double- } \\
\text {-blind RCT }\end{array}$ & $\begin{array}{l}\text { Patients randomized to receive } \\
250 \mathrm{~mL} \text { either HSD or Ringer's } \\
\text { lactate as prehospital } \\
\text { resuscitation }\end{array}$ & 211 & $\begin{array}{l}34 \pm \\
\pm 12\end{array}$ & $\begin{array}{c}184 \\
(87.2 \%)\end{array}$ & 211 & $\begin{array}{l}33 \pm \\
\pm 12\end{array}$ & $\begin{array}{c}175 \\
(82.9 \%)\end{array}$ \\
\hline $\begin{array}{l}\text { Morrison } \\
\text { et al. } 2011\end{array}$ & Canada & $\begin{array}{l}\text { Random- } \\
\text { ized con- } \\
\text { trolled } \\
\text { feasibility } \\
\text { trial }\end{array}$ & $\begin{array}{l}250 \mathrm{~mL} \text { of NS or } 250 \mathrm{~mL} \text { of HSD } \\
\text { in a single dose. If the paramed- } \\
\text { ics failed to obtain an intravenous } \\
\text { access, the study's solution could } \\
\text { be started immediately at the } \\
\text { arrival to the emergency depart- } \\
\text { ment as long as this occurred } \\
\text { within } 4 \mathrm{~h} \text { from the injury }\end{array}$ & 50 & $\begin{array}{l}46 \pm \\
\pm 21\end{array}$ & $\begin{array}{c}30 \\
(60.0 \%)\end{array}$ & 57 & $\begin{array}{l}43 \pm \\
\pm 21\end{array}$ & $\begin{array}{c}43 \\
(75.4 \%)\end{array}$ \\
\hline $\begin{array}{l}\text { Rizoli et al. } \\
2006\end{array}$ & Canada & $\begin{array}{l}\text { Double- } \\
\text {-blind } \\
\text { RCT }\end{array}$ & $\begin{array}{l}\text { Patients randomized to receive } \\
\text { a single } 250 \text {-mL bolus of either } \\
\text { HSD or normal saline. Mean } \\
\text { (standard deviation) total volume } \\
\text { in first } 24 \mathrm{~h} \text {; Control group: col- } \\
\text { loid } 696 \text { (773) } \mathrm{mL} \text {, crystalloid } 8080 \\
\text { (2736) } \mathrm{mL} \text {; HSD group: colloid } \\
361(377) \mathrm{mL} \text {, crystalloid } 7796 \\
\text { (3189) } \mathrm{mL} \text {; } \mathrm{p}=0.02 \text { and } \mathrm{p}=0.75 \\
\text { between groups for crystalloid } \\
\text { and colloid respectively }\end{array}$ & 10 & $\begin{array}{l}49.3 \pm \\
\pm 16.7\end{array}$ & $\begin{array}{c}7 \\
(70.0 \%)\end{array}$ & 14 & $\begin{array}{l}47.5 \pm \\
\pm 15.9\end{array}$ & $\begin{array}{c}9 \\
(64.3 \%)\end{array}$ \\
\hline $\begin{array}{l}\text { Vassar et al. } \\
1991\end{array}$ & USA & $\begin{array}{l}\text { Double- } \\
\text {-blind RCT }\end{array}$ & $\begin{array}{l}\text { Trauma patient were given } 250 \mathrm{~mL} \\
\text { of } 7.5 \mathrm{HSD} 70 \text { or Ringer's lactate } \\
\text { as prehospital resuscitation }\end{array}$ & 83 & $\begin{array}{c}30.3 \pm \\
\pm 6.1\end{array}$ & NS & 83 & $\begin{array}{c}32.3 \pm \\
\pm 6.1\end{array}$ & ns \\
\hline $\begin{array}{l}\text { Vassar et al. } \\
1993(1)\end{array}$ & USA & $\begin{array}{l}\text { Double- } \\
\text {-blind RCT }\end{array}$ & $\begin{array}{l}\text { Trauma patients in prehospital } \\
\text { transport were given } 250 \mathrm{~mL} \text { of: } \\
\text { (1) normal saline; (2) } 7.5 \% \mathrm{NaCl} \\
\text { (HS); (3) } 7.5 \% \mathrm{NaCl} \text { in } 6 \% \mathrm{HSD} 70\end{array}$ & 174 & $\begin{array}{l}31.5 \pm \\
\pm 14.5\end{array}$ & NS & 84 & $\begin{array}{l}31 \pm \\
\pm 12\end{array}$ & ns \\
\hline
\end{tabular}


Table 1 (cont.). Characteristics of included studies.

\begin{tabular}{|c|c|c|c|c|c|c|c|c|c|}
\hline \multirow[t]{2}{*}{ Study } & \multirow[t]{2}{*}{ Country } & \multirow[t]{2}{*}{$\begin{array}{l}\text { Study } \\
\text { design }\end{array}$} & \multirow[t]{2}{*}{ Intervention } & \multicolumn{3}{|c|}{$\begin{array}{l}\text { Hyper-saline } \\
\text { group }\end{array}$} & \multicolumn{3}{|c|}{$\begin{array}{l}\text { Control } \\
\text { group }\end{array}$} \\
\hline & & & & $\mathbf{N}$ & Age & Males & $\mathbf{N}$ & Age & Males \\
\hline $\begin{array}{l}\text { Vassar et al. } \\
1993(2)\end{array}$ & USA & $\begin{array}{l}\text { Double- } \\
\text {-blind RCT }\end{array}$ & $\begin{array}{l}\text { Trauma patients were given } \\
200 \mathrm{~mL} \text { or more of: (1) Lactate } \\
\text { Ringer's solution, (2) 7.5\% hyper- } \\
\text { tonic saline solution, (3) } 7.5 \% \text { HS } \\
\text { combined with } 6 \% \text { HSD } 70 \text {, (4) } 7.5 \\
\text { HS combined with } 12 \% \text { HSD } 70\end{array}$ & 149 & $\begin{array}{l}32 \pm \\
\pm 13\end{array}$ & NS & 45 & $\begin{array}{l}37 \pm \\
\pm 18\end{array}$ & ns \\
\hline $\begin{array}{l}\text { Wade et al. } \\
2003\end{array}$ & USA & $\begin{array}{l}\text { Double- } \\
\text {-blind RCT }\end{array}$ & $\begin{array}{c}\text { Trauma patients were given } \\
250 \mathrm{~mL} \text { of } \mathrm{HSD}(7.5 \% \mathrm{NaCl} / 6 \% \\
\text { HSD } 70) \text { or } 250 \mathrm{~mL} \text { of normal } \\
\text { saline }(0.9 \% \mathrm{NaCl})\end{array}$ & 120 & $\begin{array}{c}32 \pm \\
\pm 10.4\end{array}$ & NS & 110 & $\begin{array}{c}32 \pm \\
\pm 10.5\end{array}$ & ns \\
\hline $\begin{array}{l}\text { Younes } \\
\text { et al. } 1992\end{array}$ & Brazil & $\begin{array}{l}\text { Double- } \\
\text {-blind RCT }\end{array}$ & $\begin{array}{l}\text { Emergency unit patients received } \\
\text { either an intravenous bolus infu- } \\
\text { sion of } 250 \mathrm{~mL} \text { of hypertonic/ } \\
\text { /hypertonic } 7.5 \% \mathrm{NaCl}+6 \% \mathrm{HSD} \\
70 \text { or an isotonic } 0.9 \% \mathrm{NaCl} \text { (NS) } \\
\text { solution }\end{array}$ & 70 & NS & NS & 35 & NS & ns \\
\hline $\begin{array}{l}\text { Younes } \\
\text { et al. } 2002\end{array}$ & Brazil & $\begin{array}{l}\text { Double- } \\
\text {-blind } \\
\text { RCT }\end{array}$ & $\begin{array}{l}\text { Emergency unit patients received } \\
\text { either an intravenous bolus infu- } \\
\text { sion of } 250 \mathrm{~mL} \text { of hypertonic/ } \\
\text { /hypertonic } 7.5 \% \mathrm{NaCl}+6 \% \mathrm{HSD} \\
70 \text { or an isotonic } 0.9 \% \mathrm{NaCl} \text { (IS) } \\
\text { solution }\end{array}$ & 101 & $\begin{array}{l}39.8 \pm \\
\pm 11.2\end{array}$ & $\begin{array}{c}93 \\
(92.1 \%)\end{array}$ & 111 & $\begin{array}{l}40.8 \pm \\
\pm 12.2\end{array}$ & $\begin{array}{c}92 \\
(82.9 \%)\end{array}$ \\
\hline
\end{tabular}

HS — hypertonic saline; HSD — hypertonic saline/dextran; NS — normotonic/isotonic fluid; ns — not specified; RCT — randomized controlled trial

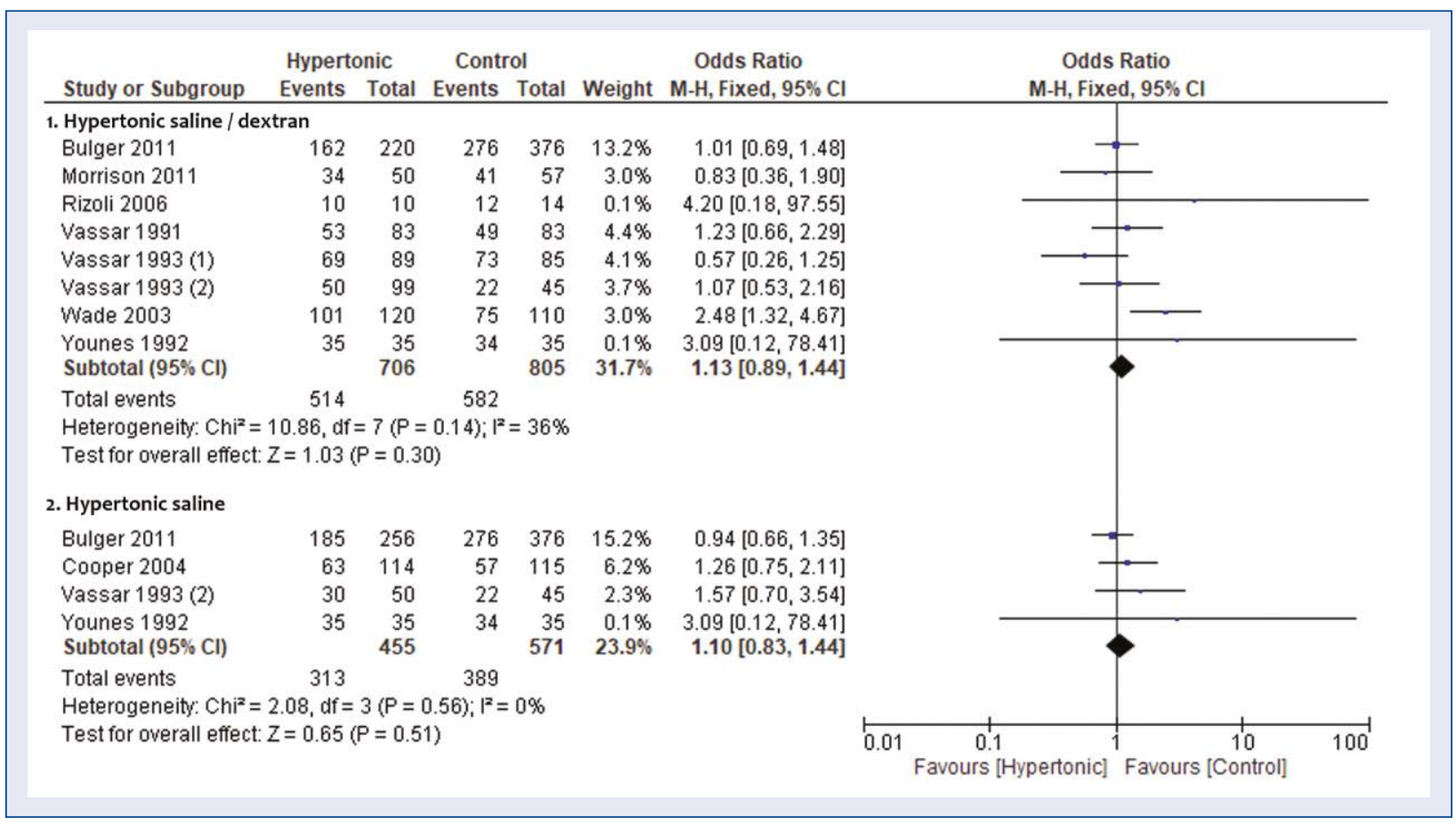

Figure 2. Forest plot of survival to hospital discharge rate while using hypertonic fluid solutions versus isotonic fluid solutions. The center of each square represents the weighted mean difference for individual trials, and the corresponding horizontal line stands for a $95 \%$ confidence interval $(\mathrm{Cl})$. The diamonds represent pooled results. 


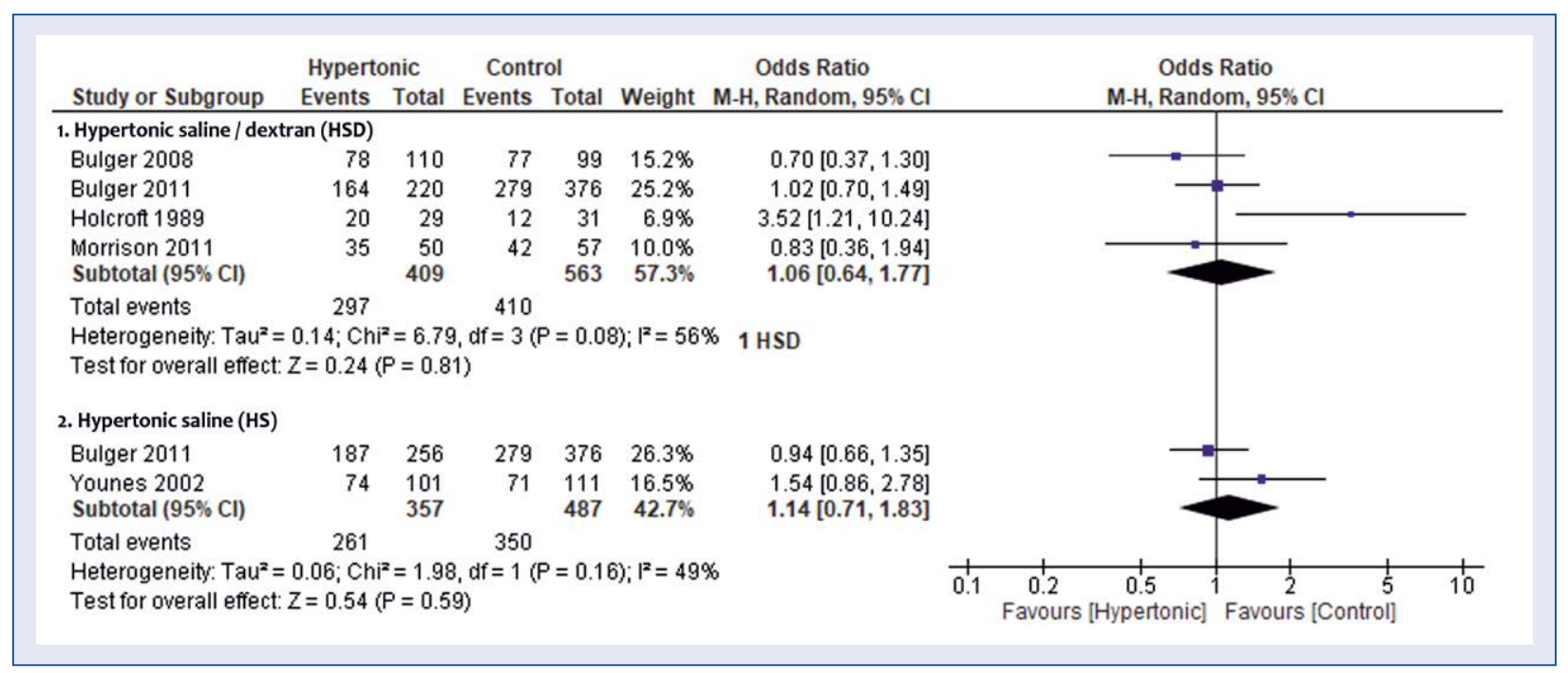

Figure 3. Forest plot of 28- to 30-days survival rate while using hypertonic fluid solutions versus isotonic fluid solutions. The center of each square represents the weighted mean difference for individual trials, and the corresponding horizontal line stands for a $95 \%$ confidence interval (CI). The diamonds represent pooled results.

\section{Secondary endpoints}

The detailed results of the secondary endpoints are presented in Table $2.24 \mathrm{~h}$ survival rate in case of hypertonic fluids was $88.6 \%$ and was higher than with isotonic fluids $72.3 \%\left(\mathrm{OR}=2.99 ; 95 \%\right.$ CI 2.04-4.39; $\mathrm{I}^{2}=0 \%$; $\mathrm{p}<0.001)$. In the case of the 3 -month survival rate, there was no significant statistical difference $(55.3 \%$ vs. $48.2 \%$; OR $=1.33 ; 95 \% \mathrm{CI}$ $0.79-2.23 ; \mathrm{p}=0.29)$.

Seven studies [14, 16, 20, 22, 26-28] reported overall mortality in the experimental group was $19.7 \%$ compared with NS group $-24.8 \%$ (OR $=0.76 ; 95 \%$ CI $\left.0.61-0.94 ; \mathrm{I}^{2}=33 \% ; \mathrm{p}=0.01\right)$. Subgroup analysis showed higher total mortality in the HSD group (23.3\% for hypertonic vs. $17.3 \%$ for isotonic group; $\mathrm{p}=0.01$ ) as well as in the hypertonic saline group (25.9\% vs. $23.7 \%$, respectively; $\mathrm{p}=0.51$; Fig. 4 ).

The use of hypertonic fluid was associated with a longer hospital stay than with isotonic fluid solutions (mean difference $[\mathrm{MD}]=1.45 ; 95 \% \mathrm{CI}$ $0.43-2.46 ; \mathrm{p}=0.005)$. Acute respiratory distress syndrome-free survival rate at 28 days was reported in 2 studies. The difference between hypersaline and normosaline groups was not statistically significant $(\mathrm{OR}=1.10 ; 95 \%$ CI $0.85-1.44$; $\mathrm{p}=0.46)$.

The use of hypertonic fluid solutions was associated with higher SBP at hospital admission compared to isotonic fluids $(\mathrm{MD}=6.71 ; 95 \% \mathrm{CI}$
1.75-11.67; $\mathrm{I}^{2}=72 \% ; \mathrm{p}=0.008$; Suppl. Digital File 1).

Polled analyses illustrated selected laboratory parameters are presented in Supplementary Digital File 1.

\section{Adverse events}

Pooled analysis showed no statistically significant incidence of complications between hypertonic vs. isotonic fluids solutions. Detailed analysis of particular types of adverse events is presented in Table 3. The most frequently observed nosocomial infections were pneumonia, urinary tract infection, or bloodstream infection. For noninfectious complications: abdominal compartment syndrome, cerebral infarction, or deep vein thrombosis. A summary of the injuries related and the use of fluid types is presented in Supplementary Digital File 1.

\section{Publication bias}

The risk of bias of all the RCTs included in the meta-analysis is shown in Supplementary Digital File 1. Overall, the included RCTs suggested good quality in terms of risk of bias.

\section{Discussion}

This systematic review and meta-analysis evaluated data from RCTs of hypertonic fluid solutions (HSD or hypertonic saline) and isotonic 
Table 2. Characteristics of outcomes: hypertonic fluid solutions versus isotonic fluid solutions.

\begin{tabular}{|c|c|c|c|c|c|c|c|}
\hline \multirow[t]{3}{*}{$\begin{array}{l}\text { Type of adverse } \\
\text { event }\end{array}$} & \multirow[t]{3}{*}{$\begin{array}{l}\text { Number } \\
\text { of trials }\end{array}$} & \multirow{3}{*}{$\begin{array}{c}\text { Total } \\
\text { numer } \\
\text { of patients }\end{array}$} & \multicolumn{2}{|c|}{$\begin{array}{l}\text { Percentage of } \\
\text { adverse event }\end{array}$} & \multirow{3}{*}{$\begin{array}{l}\text { Treatment effect } \\
\text { (hypertonic vs. } \\
\text { normotonic fluid } \\
\text { solutions) } \\
\text { OR/MD (95\% CI) }\end{array}$} & \multirow[t]{3}{*}{ P value } & \multirow{3}{*}{$\begin{array}{c}\mathrm{I}^{2}, \\
\text { statistic, } \\
\%\end{array}$} \\
\hline & & & \multirow{2}{*}{$\begin{array}{l}\text { HSD } \\
\text { or HS }\end{array}$} & \multirow[t]{2}{*}{ NS } & & & \\
\hline & & & & & & & \\
\hline \multicolumn{8}{|l|}{ 24-h survival } \\
\hline HSD & 2 & 575 & $89.4 \%$ & $73.8 \%$ & 2.99 (1.88-4.75) & $<0.001$ & $18 \%$ \\
\hline NS & 2 & 332 & $86.4 \%$ & $68.6 \%$ & $3.01(1.54-5.89)$ & 0.001 & $23 \%$ \\
\hline Total & 4 & 807 & $88.6 \%$ & $72.3 \%$ & $2.99(2.04-4.39)$ & $<0.001$ & $0 \%$ \\
\hline \multicolumn{8}{|c|}{ 28- to 30 -day survival } \\
\hline HSD & 4 & 972 & $72.6 \%$ & $72.8 \%$ & $1.06(0.64-1.77)$ & 0.81 & $56 \%$ \\
\hline NS & 2 & 844 & $73.1 \%$ & $71.9 \%$ & $1.14(0.71-1.83)$ & 0.59 & $49 \%$ \\
\hline Total & 5 & 1440 & $72.8 \%$ & $71.4 \%$ & $1.13(0.75-1.70)$ & 0.56 & $54 \%$ \\
\hline \multicolumn{8}{|c|}{ Survival to discharge } \\
\hline HSD & 8 & 1511 & $72.8 \%$ & $72.3 \%$ & $1.13(0.89-1.44)$ & 0.30 & $36 \%$ \\
\hline NS & 4 & 1026 & $68.8 \%$ & $68.1 \%$ & $1.10(0.83-1.44)$ & 0.51 & $0 \%$ \\
\hline Total & 9 & 2081 & $71.2 \%$ & $69.4 \%$ & $1.19(0.97-1.45)$ & 0.09 & $48 \%$ \\
\hline \multicolumn{8}{|c|}{ Survival at 3 months } \\
\hline HSD & - & - & - & - & - & - & - \\
\hline NS & 1 & 228 & $55.3 \%$ & $48.2 \%$ & $1.33(0.79-2.23)$ & 0.29 & - \\
\hline Total & 1 & 228 & $55.3 \%$ & $48.2 \%$ & $1.33(0.79-2.23)$ & 0.29 & - \\
\hline \multicolumn{8}{|c|}{ Length of hospital stay (days) } \\
\hline HSD & 3 & 361 & - & - & $1.05(-1.88-3.98)$ & 0.48 & $0 \%$ \\
\hline NS & 1 & 222 & - & - & $1.50(0.42-2.58)$ & 0.007 & - \\
\hline Total & 4 & 583 & - & - & $1.45(0.43-2.46)$ & 0.005 & $0 \%$ \\
\hline \multicolumn{8}{|c|}{ ARDS-free survival rate to day 28} \\
\hline HSD & 2 & 805 & $51.8 \%$ & $54.9 \%$ & $1.15(0.84-1.57)$ & 0.39 & $0 \%$ \\
\hline NS & 1 & 632 & $66.0 \%$ & $65.4 \%$ & $1.03(0.73-1.43)$ & 0.88 & - \\
\hline Total & 2 & 1061 & $58.0 \%$ & $54.9 \%$ & $1.10(0.85-1.44)$ & 0.46 & $6 \%$ \\
\hline \multicolumn{8}{|c|}{ Total fluids in first $24-\mathrm{h}$} \\
\hline HSD & 4 & 1268 & - & - & $-1.14(-2.15-0.13)$ & 0.03 & $0 \%$ \\
\hline NS & 1 & 632 & - & - & $-0.70(-2.47-1.07)$ & 0.44 & - \\
\hline Total & 4 & 1524 & - & - & $-1.07(-2.03-0.12)$ & 0.03 & $0 \%$ \\
\hline \multicolumn{8}{|c|}{ Hypernatremia ( $\mathrm{Na}>160 \mathrm{mEq} / \mathrm{L}$ ) requiring intervention } \\
\hline HSD & 1 & 596 & $0.9 \%$ & $1.3 \%$ & $0.68(0.13-3.54)$ & 0.65 & - \\
\hline NS & 1 & 632 & $1.9 \%$ & $1.3 \%$ & $1.48(0.42-5.16)$ & 0.54 & - \\
\hline Total & 1 & 852 & $1.5 \%$ & $1.3 \%$ & $1.11(0.35-3.52)$ & 0.86 & - \\
\hline \multicolumn{8}{|l|}{ Overall mortality } \\
\hline HSD & 6 & 1459 & $17.3 \%$ & $23.3 \%$ & $0.72(0.55-0.94)$ & 0.01 & $20 \%$ \\
\hline NS & 3 & 914 & $23.7 \%$ & $25.9 \%$ & $0.90(0.66-1.23)$ & 0.51 & $10 \%$ \\
\hline Total & 7 & 1962 & $19.7 \%$ & $24.8 \%$ & $0.76(0.61-0.94)$ & 0.01 & $33 \%$ \\
\hline
\end{tabular}

ARDS - acute respiratory distress syndrome; $\mathrm{Cl}$ - confidence interval; $\mathrm{HSD}$ - hypertonic fluid solutions; MD — mean difference; NS - izotonic/norotonic fluid solutions; OR - odds ratio

fluid solutions $(0.9 \% \mathrm{NaCl}$ or lactated Ringer's solution) for fluid resuscitation in fluid with traumatic hypovolemic shock, encompassing 15 studies and approximately 3264 adult trauma patients. At primary timepoints assessed (including at 28- to 30 -days survival rate or survival to hospital dis- 


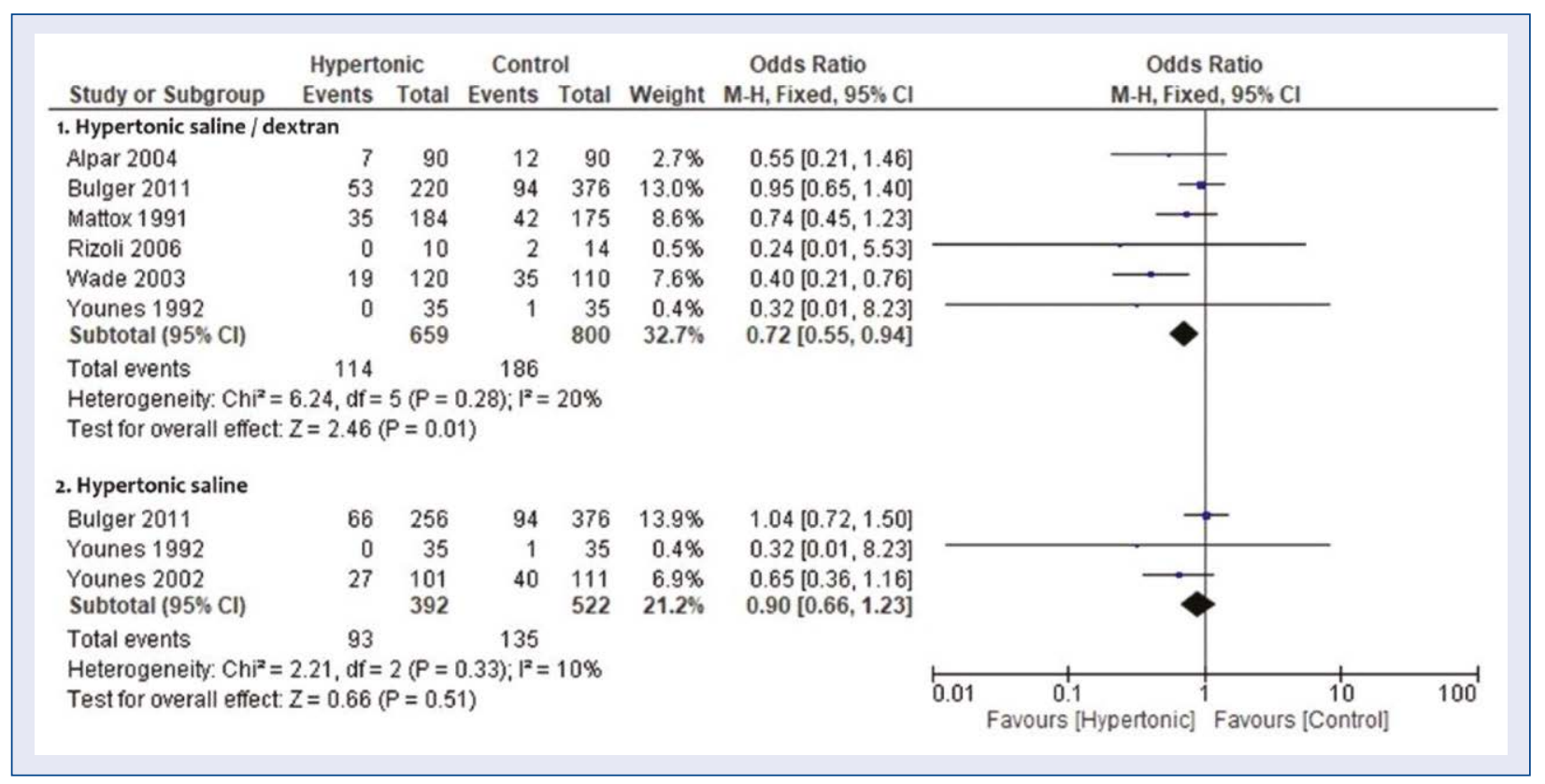

Figure 4. Forest plot of overall mortality rate while using hypertonic fluid solutions versus isotonic fluid solutions. The center of each square represents the weighted mean difference for individual trials, and the corresponding horizontal line stands for a $95 \%$ confidence interval $(\mathrm{Cl})$. The diamonds represent pooled results.

charge), treatment with hypertonic fluid solutions was associated with a higher rate than treatment with isotonic fluid solutions). However, in the case of 24-survival rate treatment with hypertonic fluid solutions was related to a significantly higher survival rate, as well as significantly lower overall mortality.

$0.9 \%$ sodium chloride solution is a basic crystalline fluid used in both pre-hospital and hospital care [29]. Due to high chlorine levels in the isotonic salt, there is a potential risk of metabolic hyperchloremic acidosis [30]. An alternative to $0.9 \%$ $\mathrm{NaCl}$ is Ringer's lactate, also called Hartman's solution, where the sodium and calcium concentration corresponds to the plasma concentration of these ions. However, it is important to note that the calcium contained in Ringer's lactate can bind to and interfere with some drugs. Indeed, Ringer's lactate is not free of disadvantages. Its main disadvantage is that it binds calcium to citrate anticoagulants in blood products, which can lead to clots in the bloodstream. Due to the above, Ringer's lactate is contraindicated as a diluent for blood transfusions [31, 32]. Hypertonic solutions, on the other hand, result in a slight improvement in volume and a rapid restoration of hemodynamics. The present analysis looked at hemodynamic parameters, such as SBP, and indicated that the use of hypertonic fluid solutions was associated with a statistically significant higher SBP than that of isotonic solutions $(\mathrm{p}=0.008)$.

According to laboratory studies, hypertonic solutions especially improve the hemodynamics of microcirculation. This is due to the recruitment of intra-tissue volume by these fluids, which increases the volume of circulating blood and at the same time increases blood pressure. According to numerous studies, a $7.5 \% \mathrm{NaCl}$ solution should be administered at $250 \mathrm{~mL}$ or $4 \mathrm{~mL} / \mathrm{kg}$ body weight [33]. The same volume of hypertonic fluid administered compared to the isotonic solution causes a greater increase in the volume of fluid in the vascular bed, as this difference comes from the intracellular fluid, which penetrates from the cells into the extracellular space. Therefore, the use of hypertonic solutions should be reflected in the treatment of trauma patients as they allow to restore intravascular volume without increasing intravascular space [34]. Moreover, the present results showed no significant differences in adverse events between the treatment of hypertonic fluid solutions compared with isotonic solutions. However, it should be noted that many studies have not reported adverse events, which is a potential source of bias.

\section{Limitations of the study}

There are potential limitations in this systematic review and meta-analysis. One limitation is to 
Table 3. Characteristics of adverse events between hypertonic fluid solutions versus isotonic fluid solutions.

\begin{tabular}{|c|c|c|c|c|c|c|c|}
\hline \multirow[t]{2}{*}{ Type of adverse event } & \multirow{2}{*}{$\begin{array}{l}\text { Number } \\
\text { of trials }\end{array}$} & \multirow{2}{*}{$\begin{array}{c}\text { Total } \\
\text { numer of } \\
\text { patients }\end{array}$} & \multicolumn{2}{|c|}{$\begin{array}{l}\text { Percentage of } \\
\text { adverse event }\end{array}$} & \multirow[t]{2}{*}{$\begin{array}{c}\text { OR } \\
(95 \% \mathrm{CI})\end{array}$} & \multirow[t]{2}{*}{$\begin{array}{c}P \\
\text { value }\end{array}$} & \multirow{2}{*}{$\begin{array}{c}\mathrm{I}^{2}, \\
\text { statistic, } \\
\%\end{array}$} \\
\hline & & & $\begin{array}{l}\text { HSD } \\
\text { or HS }\end{array}$ & NS & & & \\
\hline \multicolumn{8}{|l|}{ Nosocomial inections } \\
\hline Pneumonia & 4 & 1695 & $9.9 \%$ & $9.8 \%$ & $\begin{array}{c}0.95 \\
(0.68-1.31)\end{array}$ & 0.75 & $0 \%$ \\
\hline ARDS & 1 & 422 & $0.0 \%$ & $0.9 \%$ & $\begin{array}{c}0.20 \\
(0.01-4.15)\end{array}$ & 0.30 & - \\
\hline Blood stream infection & 2 & 1061 & $7.2 \%$ & $6.1 \%$ & $\begin{array}{c}1.18 \\
(0.72-1.93)\end{array}$ & 0.51 & $0 \%$ \\
\hline Urinary tract infection & 2 & 1061 & $6.1 \%$ & $7.6 \%$ & $\begin{array}{c}0.79 \\
(0.49-1.28)\end{array}$ & 0.34 & $0 \%$ \\
\hline Wound infection & 2 & 1061 & $5.8 \%$ & $4.0 \%$ & $\begin{array}{c}1.50 \\
(0.84-2.67)\end{array}$ & 0.17 & $0 \%$ \\
\hline Intra-abdominal abcess & 2 & 631 & $1.6 \%$ & $0.3 \%$ & $\begin{array}{c}3.49 \\
(0.57-21.54)\end{array}$ & 0.18 & $11 \%$ \\
\hline Sinustis & 1 & 209 & $0.9 \%$ & $0.0 \%$ & $\begin{array}{c}2.71 \\
(0.11-67.69)\end{array}$ & 0.54 & - \\
\hline Pseudomembranous colitis & 1 & 209 & $1.0 \%$ & $0.0 \%$ & $\begin{array}{c}2.73 \\
(0.11-67.69)\end{array}$ & 0.54 & - \\
\hline Line infection & 1 & 209 & $1.0 \%$ & $0.0 \%$ & $\begin{array}{c}2.73 \\
(0.11-67.69)\end{array}$ & 0.54 & - \\
\hline Sepsis & 1 & 422 & $0.0 \%$ & $1.4 \%$ & $\begin{array}{c}0.14 \\
(0.01-2.74)\end{array}$ & 0.20 & - \\
\hline Other & 1 & 311 & $3.8 \%$ & $0.0 \%$ & $\begin{array}{c}8.27 \\
(0.47-144.75)\end{array}$ & 0.15 & - \\
\hline One or more nosocomial infections & 2 & 1061 & $23.0 \%$ & $21.9 \%$ & $\begin{array}{c}1.06 \\
(0.79-1.42)\end{array}$ & 0.70 & $0 \%$ \\
\hline \multicolumn{8}{|l|}{ Noninfectious complications } \\
\hline Acute renal failure & 3 & 780 & $0.8 \%$ & $1.6 \%$ & $\begin{array}{c}0.52 \\
(0.14-1.95)\end{array}$ & 0.33 & $0 \%$ \\
\hline Abdominal compartment syndrome & 1 & 209 & $3.6 \%$ & $8.1 \%$ & $\begin{array}{c}0.43 \\
(0.13-1.47)\end{array}$ & 0.18 & - \\
\hline Cardiac arrest & 2 & 568 & $1.0 \%$ & $1.5 \%$ & $\begin{array}{c}0.71 \\
(0.17-2.88)\end{array}$ & 0.63 & \\
\hline Myocardial infarction & 3 & 780 & $1.0 \%$ & $2.1 \%$ & $\begin{array}{c}0.52 \\
(0.16-1.67)\end{array}$ & 0.28 & $0 \%$ \\
\hline Cerebral infarction & 2 & 421 & $4.3 \%$ & $2.8 \%$ & $\begin{array}{c}1.61 \\
(0.58-4.53)\end{array}$ & 0.36 & $0 \%$ \\
\hline Dead bowel & 1 & 359 & $0.0 \%$ & $0.6 \%$ & $\begin{array}{c}0.32 \\
(0.01-7.79)\end{array}$ & 0.48 & - \\
\hline Deep vein thrombolysis & 1 & 209 & $0.9 \%$ & $7.0 \%$ & $\begin{array}{c}0.12 \\
(0.01-1.00)\end{array}$ & 0.05 & - \\
\hline Pulmonary embolism & 2 & 568 & $0.3 \%$ & $1.1 \%$ & $\begin{array}{c}0.39 \\
(0.06-2.70)\end{array}$ & 0.34 & $0 \%$ \\
\hline Coagulopathy & 1 & 359 & $0.9 \%$ & $0.0 \%$ & $\begin{array}{c}2.73 \\
(0.11-67.69)\end{array}$ & 0.54 & - \\
\hline
\end{tabular}

ARDS - acute respiratory distress syndrome; $\mathrm{Cl}$ - confidence interval; HS — hypertonic saline; HSD — hypertonic fluid solutions; $\mathrm{NS}$ - isotonic/norotonic fluid solutions; OR - odds ratio 
include only studies on the use of fluid therapy in patients with hypovolemic shock resulting from the injury. However, this was deliberate because it is a specific group of patients who require different treatment from patients with no hypovolemic shock due to the bleeding. The second limitation of the study is the fact that over the last years no randomized study has been published in the scope discussed in the article. With the development of medical technology and the creation of new guidelines of conduct, the authors believe that a multi-center study should be carried out, involving a large number of patients, which would verify the data from previous articles.

\section{Conclusions}

This systematic review and meta-analysis, which included only evidence from RCTs hypertonic saline/dextran or hypertonic saline compared with isotonic fluid did not result in superior 28- to 30-day survival as well as in survival to hospital discharge. However, patients with hypotension who received resuscitation with HSD had less overall mortality than patients who received conventional fluid. These findings highlight an urgent need for further research and guidance for physicians regarding when to administer fluid solutions to ensure optimal fluid therapy for the resuscitation of hypovolemic shock caused by acute hemorrhage.

\section{Acknowledgments}

The study was supported by the ERC Research Net and by the Polish Society of Disaster Medicine.

\section{Conflict of interest: None declared}

\section{References}

1. Kelley DM. Hypovolemic shock: an overview. Crit Care Nurs Q. 2005; 28(1): 2-19; quiz 20, doi: 10.1097/00002727-20050100000002, indexed in Pubmed: 15732421.

2. Kobayashi L, Costantini TW, Coimbra R. Hypovolemic shock resuscitation. Surg Clin North Am. 2012; 92(6): 1403-1423, doi: 10.1016/j.suc.2012.08.006, indexed in Pubmed: 23153876.

3. Dolanbay T, Aksoy N, Gul H, et al. Evaluation of paediatric blunt abdomen trauma patients presenting to the emergency room. Disaster Emerg Med J. 2020; 5(1): 19-23, doi: 10.5603/demj. a2020.0006.

4. Annane D, Siami S, Jaber S, et al. Effects of fluid resuscitation with colloids vs crystalloids on mortality in critically ill patients presenting with hypovolemic shock: the CRISTAL randomized trial. JAMA. 2013; 310(17): 1809-1817, doi: 10.1001/ jama.2013.280502, indexed in Pubmed: 24108515.

5. Galvagno SM, Nahmias JT, Young DA. Advanced trauma life support update 2019: management and applications for adults and special populations. Anesthesiol Clin. 2019; 37(1): 13-32, doi: 10.1016/j.anclin.2018.09.009, indexed in Pubmed: 30711226.

6. Simma B, Burger R, Falk M, et al. A prospective, randomized, and controlled study of fluid management in children with severe head injury: lactated Ringer's solution versus hypertonic saline. Crit Care Med. 1998; 26(7): 1265-1270, doi: 10.1097/00003246199807000-00032, indexed in Pubmed: 9671379.

7. Maguigan KL, Dennis BM, Hamblin SE, et al. Method of hypertonic saline administration: effects on osmolality in traumatic brain injury patients. J Clin Neurosci. 2017; 39: 147-150, doi: 10.1016/j.jocn.2017.01.025, indexed in Pubmed: 28215427.

8. Reddy S, Weinberg L, Young P. Crystalloid fluid therapy. Crit Care. 2016; 20: 59, doi: 10.1186/s13054-016-1217-5, indexed in Pubmed: 26976277.

9. Martin GS, Bassett P. Crystalloids vs. colloids for fluid resuscitation in the Intensive Care Unit: A systematic review and meta-analysis. J Crit Care. 2019; 50: 144-154, doi: 10.1016/j. jcrc.2018.11.031, indexed in Pubmed: 30540968.

10. Safiejko K, Smereka J, Filipiak KJ, et al. Effectiveness and safety of hypotension fluid resuscitation in traumatic hemorrhagic shock: a systematic review and meta-analysis of randomized controlled trials. Cardiol J. 2020 [Epub ahead of print], doi: 10.5603/ CJ.a2020.0096, indexed in Pubmed: 32648249.

11. Hutton B, Salanti G, Caldwell DM, et al. The PRISMA extension statement for reporting of systematic reviews incorporating network meta-analyses of health care interventions: checklist and explanations. Ann Intern Med. 2015; 162(11): 777-784, doi: 10.7326/M14-2385, indexed in Pubmed: 26030634.

12. Higgins J, Green S, (editors). Cochrane Handbook for Systematic Reviews of Interventions Version 5.1.0. The Cochrane Collaboration 2011. www.cochrane-handbook.org (updated March 2011).

13. Hozo SP, Djulbegovic B, Hozo I. Estimating the mean and variance from the median, range, and the size of a sample. BMC Med Res Methodol. 2005; 5: 13, doi: 10.1186/1471-2288-5-13, indexed in Pubmed: 15840177.

14. Alpar EK, Killampalli VV. Effects of hypertonic dextran in hypovolaemic shock: a prospective clinical trial. Injury. 2004; 35(5): 500-506, doi: 10.1016/S0020-1383(03)00196-7, indexed in Pubmed: 15081328 .

15. Bulger EM, Jurkovich GJ, Nathens AB, et al. Hypertonic resuscitation of hypovolemic shock after blunt trauma: a randomized controlled trial. Arch Surg. 2008; 143(2): 139-48; discussion 149, doi: 10.1001/archsurg.2007.41, indexed in Pubmed: 18283138.

16. Bulger EM, May S, Kerby JD, et al. Out-of-hospital hypertonic resuscitation after traumatic hypovolemic shock: a randomized, placebo controlled trial. Ann Surg. 2011; 253(3): 431-441, doi: 10.1097/SLA.0b013e3181fcdb22, indexed in Pubmed: 21178763.

17. Cooper DJ, Myles PS, McDermott FT, et al. Prehospital hypertonic saline resuscitation of patients with hypotension and severe traumatic brain injury: a randomized controlled trial. JAMA. 2004; 291(11): 1350-1357, doi: 10.1001/jama.291.11.1350, indexed in Pubmed: 15026402.

18. Holcroft JW, Vassar MJ, Turner JE, et al. $3 \% \mathrm{NaCl}$ and $7.5 \% \mathrm{NaCl} /$ dextran 70 in the resuscitation of severely injured patients. Ann Surg. 1987; 206(3): 279-288, doi: 10.1097/00000658-19870900000006, indexed in Pubmed: 2443087.

19. Holcroft JW, Vassar MJ, Perry CA, et al. Use of a $7.5 \% \mathrm{NaCl} / 6 \%$ Dextran 70 solution in the resuscitation of injured patients in the emergency room. Prog Clin Biol Res. 1989; 299: 331-338, indexed in Pubmed: 2471213.

20. Mattox KL, Maningas PA, Moore EE, et al. Prehospital hypertonic saline/dextran infusion for post-traumatic hypotension. The 
U.S.A. Multicenter Trial. Ann Surg. 1991; 213(5): 482-491, doi: 10.1097/00000658-199105000-00014, indexed in Pubmed: 1708984.

21. Morrison LJ, Baker AJ, Rhind SG, et al. The Toronto prehospital hypertonic resuscitation--head injury and multiorgan dysfunction trial: feasibility study of a randomized controlled trial. J Crit Care. 2011; 26(4): 363-372, doi: 10.1016/j.jcrc.2010.08.021, indexed in Pubmed: 21106341.

22. Rizoli SB, Rhind SG, Shek PN, et al. The immunomodulatory effects of hypertonic saline resuscitation in patients sustaining traumatic hemorrhagic shock: a randomized, controlled, doubleblinded trial. Ann Surg. 2006; 243(1): 47-57, doi: 10.1097/01. sla.0000193608.93127.b1, indexed in Pubmed: 16371736.

23. Vassar MJ, Perry CA, Gannaway WL, et al. 7.5\% sodium chloride/ dextran for resuscitation of trauma patients undergoing helicopter transport. Arch Surg. 1991; 126(9): 1065-1072, doi: 10.1001/ archsurg.1991.01410330019002, indexed in Pubmed: 1718243.

24. Vassar MJ, Fischer RP, O’Brien PE, et al. A multicenter trial for resuscitation of injured patients with $7.5 \%$ sodium chloride. The effect of added dextran 70. The Multicenter Group for the Study of Hypertonic Saline in Trauma Patients. Arch Surg. 1993; 128(9): 1003-11; discussion 1011, doi: 10.1001/archsurg.1993.01420210067009, indexed in Pubmed: 7690225.

25. Vassar M, Perry C, Holcroft J. Prehospital resuscitation of hypotensive trauma patients with $7.5 \% \mathrm{NaCl}$ versus $7.5 \% \mathrm{NaCl}$ with added dextran: a controlled trial. JTrauma. 1993; 34(5): 622-633, doi: 10.1097/00005373-199305000-00003.

26. Wade CE, Grady JJ, Kramer GC. Efficacy of hypertonic saline dextran fluid resuscitation for patients with hypotension from penetrating trauma. J Trauma. 2003; 54(5 Suppl): S144-S148, doi: 10.1097/01. TA.0000047223.62617.AB, indexed in Pubmed: 12768117.

27. Younes RN, Aun F, Accioly CQ, et al. Hypertonic solutions in the treatment of hypovolemic shock: a prospective, randomized study in patients admitted to the emergency room. Surgery. 1992; 111(4): 380-385, indexed in Pubmed: 1373007.

28. Younes RN, Birolini D. Hypertonic/hyperoncotic solution in hypovolemic patients: experience in the emergency room. Rev Hosp Clin Fac Med Sao Paulo. 2002; 57(3): 124-128, doi: 10.1590/ s0041-87812002000300008, indexed in Pubmed: 12118271.

29. Krzych ŁJ, Czempik PF. Effect of fluid resuscitation with balanced solutions on platelets: In vitro simulation of $20 \%$ volume substitution. Cardiol J. 2018; 25(2): 254-259, doi: 10.5603/ CJ.a2017.0054, indexed in Pubmed: 28497841.

30. Barker ME. $0.9 \%$ saline induced hyperchloremic acidosis. J Trauma Nurs. 2015; 22(2): 111-116, doi: 10.1097/ JTN.0000000000000115, indexed in Pubmed: 25768968.

31. Kiraly LN, Differding JA, Enomoto TM, et al. Resuscitation with normal saline (NS) vs. lactated ringers (LR) modulates hypercoagulability and leads to increased blood loss in an uncontrolled hemorrhagic shock swine model. J Trauma. 2006; 61(1): 57-64; discussion 64, doi: 10.1097/01.ta.0000220373.29743.69, indexed in Pubmed: 16832250.

32. Zitek T, Skaggs ZD, Rahbar A, et al. Does Intravenous Lactated Ringer's Solution Raise Serum Lactate? J Emerg Med. 2018; 55(3): 313-318, doi: 10.1016/j.jemermed.2018.05.031, indexed in Pubmed: 30037514.

33. Stern SA, Jwayyed S, Dronen SC, et al. Resuscitation of severe uncontrolled hemorrhage: $7.5 \%$ sodium chloride/6\% dextran 70 vs 0.9\% sodium chloride. Acad Emerg Med. 2000; 7(8): 847-856, doi: 10.1111/j.1553-2712.2000.tb02060.x, indexed in Pubmed: 10958123.

34. Søreide E, Deakin CD. Pre-hospital fluid therapy in the critically injured patient - a clinical update. Injury. 2005; 36(9): 1001-1010, doi: 10.1016/j.injury.2005.01.002, indexed in Pubmed: 16098325 . 